\title{
Design of Intelligent Public Transportation System based on ZigBee Technology
}

\author{
Jing Bian*, Xiuxia Yu, and Wei Du \\ College of Computer Science and Technology, Changchun University, Changchun, 130022, China
}

\begin{abstract}
In this article, an intelligent public transportation system based on ZigBee 3.0 technology is proposed after researching conditions of recent public transportation systems that mostly adopt GPS or Beidou satellite positioning technology, 3G/4G communication technology and GIS technology. This system includes the principle and design of system architecture, design of intelligent public transportation system network, the solution of moving-bus positioning, the auto-announce function and design of intelligent public transportation stop board. Contrasted with recent bus systems, this system has the virtue of low construction cost, low running cost, low implementation difficulty and high intelligent level. ZigBee 3.0 protocol is compatible with Wi-Fi, too. Without additional ZigBee chip in smart phones, the intelligent public transportation system based on ZigBee 3.0 can interconnect with smart phones directly. It will break the barrier between citizens and intelligent public transportation systems, and the real intelligence can be realized.
\end{abstract}

Keywords: ZigBee; intelligent public transportation; IoT; bus positioning; intelligent public transportation stop board

(Submitted on December 13, 2017; Revised on January 16, 2018; Accepted on February 9, 2018)

(C) 2018 Totem Publisher, Inc. All rights reserved.

\section{Introduction}

The intelligent public transportation systems now available mainly adopt GPS or Beidou satellite positioning technology, 3G/4G communication technology and GIS technology. But such an intelligent public transportation system is too complex to implement and is easily affected by the environment [8]. The high construction cost and high running cost make it impossible to realize in most undeveloped cities. Furthermore, the cities with mountainous areas are impossible to implement. Only a few developed large cities with advantageous geographical environment have the ability to construct this kind of intelligent public transportation system. So, the plan is hard to popularize on a large scale.

ZigBee is a kind of short-range wireless communication technology. It is a LAN protocol with low power consumption based on IEEE802.15.4 standard. Its characteristics include close range, low complexity, self-organizing, low delay, low power consumption, low cost and low data rate [7]. ZigBee is very suitable to be the basic communication technology of intelligent public transportation systems with all the advantages above. For these reasons, intelligent public transportation system based on ZigBee technology is proposed in this article. In the system, the very cheap ZigBee devices are used to communicate between the bus and the intelligent public transportation stop board, as well as between the intelligent public transportation stop boards themselves. At the same time, the RSSI (Received Signal Strength Indication) method is used to determine the distance between the signal node and the destination node according to the strength of the signals received. It can be done using the calculations of data and position of the bus so as to gain the running conditions of the bus. Then, the real intelligent public transportation system will come true. It seems obviously that the intelligent public transportation system based on ZigBee technology is easy to implement, and it is easy to popularize on a large scale with low construction and running cost. 


\section{Investigation into the intelligent public transportation system based on ZigBee}

After researching intelligent public transportation systems now available, a new kind of system is proposed in this article. It is not complex, not expensive, and is easy to popularize on a large scale. It will provide a new breakthrough for the development of intelligent public transportation systems. There are three modes for existing intelligent public transportation systems based on ZigBee.

The first mode is to make bus positioning by GPS or Beidou satellite, and make short distance wireless communication between bus and bus stop board by ZigBee technology. The communication between bus stop boards and the communication between bus stop board and bus dispatching center are implemented by a wired network. This is a mode of combining ZigBee technology with wire communication.

The second mode adopted the same positioning method, but the communication between bus stop boards and the communication between bus stop board and dispatching center are implemented by $3 \mathrm{G} / 4 \mathrm{G}$ wireless communication. In this mode, the main communication method is $3 \mathrm{G} / 4 \mathrm{G}$ and ZigBee technology only assists.

The third mode is to divide the city into several areas. In each area, a big scale of ZigBee network is settled, in which buses communicate with bus stop boards, and the bus stop boards communicate with each other. GPS or Beidou satellite is still be used to make bus positioning. Communication between two areas are implemented by ZigBee border routing nodes. Then, the information of each area is collected by bus dispatching center. This mode realizes the whole intelligent public transportation system.

The first two modes will not change much from the existing intelligent public transportation system. The first mode isn't flexible as cities that develop fast will have too many cables to be settled. The second mode will not improve either because the only change is to replace the current communication with ZigBee between the bus and bus stop board. The function of intelligent public transportation system will not be improved and the cost will not be cut down. The third mode changes more but doesn't change the positioning method, which costs the most. The running situation of buses with ZigBee completely can't be transmitted to the dispatching center in time, so the dispatching center can't act promptly.

\section{Principle and design of system architecture}

According to the problems of existing systems, a new intelligent public transportation system based on ZigBee technology is proposed in this article. In this system, ZigBee communication modules are needed to be settled on buses and bus stop boards. The architecture diagram of the intelligent public transportation system is shown in Figure 1. In this system, GPS or Beidou satellite positioning method and $3 \mathrm{G} / 4 \mathrm{G}$ communication mode are abandoned, which will reduce the construction cost and running cost greatly.

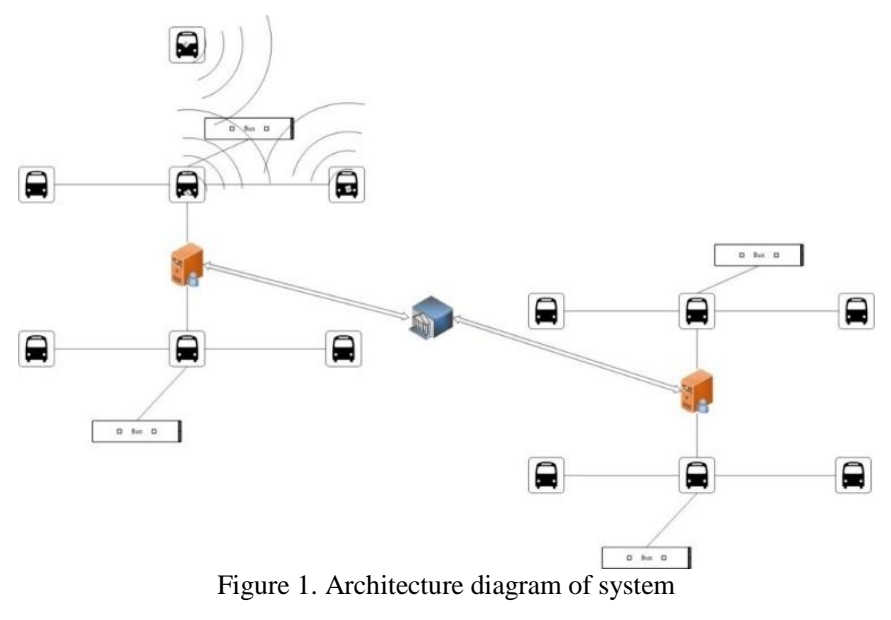

As shown in Figure 1, the thin black lines indicate ZigBee communication and the bold bidirectional arrow indicates optical fiber communication. The communication between bus and bus stop board and the one between bus stop boards are all by ZigBee technology. Some of the bus stop boards communicate with information processing center by ZigBee, and the information processing center communicates with the dispatching center by optical fiber directly. Then, the whole communication network is built. By receiving the ZigBee signal around it and according to the strength of the signal and the position information in it, the bus positions itself by the RSSI algorithm. Then, the ZigBee communication module on the bus 
sends position information to the nearest bus stop board. The bus stop board then sends the information to other ones by ZigBee communication. When the information is sent to the information processing center in the end, the information is preprocessed and gathered and then sent to the dispatching center through optical fiber. Then, the bus dispatching center can make dynamic dispatch according to the current bus running situation.

\section{Design of intelligent public transportation system network}

ZigBee devices are divided into 2 types: Full-Function Device (FFD) and Reduced- Function De-vice (RFD) [1]. Compared with FFD, RFD loads less protocols, consumes less energy, is unable to forward data, and can only be a terminal to communicate with FFD. The ZigBee equipment that is arranged on the bus does not need to forward data, so the selection of the device can be RFD. ZigBee device on the bus stop needs a lot of data forwarding, so a FFD must be chosen.

ZigBee supports three basic network topologies: Tree topology, Star topology and Mesh topology [3]. These three network topologies all require a FFD to act as a network coordinator, as shown in Figure 2. Other FFD can also be added as a router or end device.

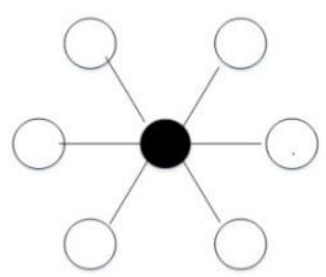

Star topology

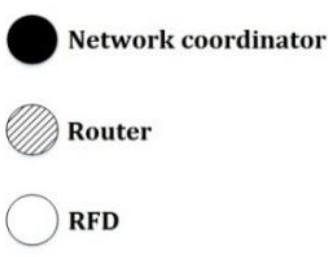

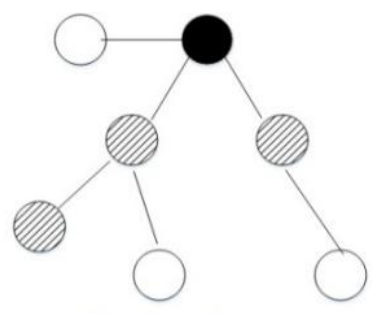

Tree topology

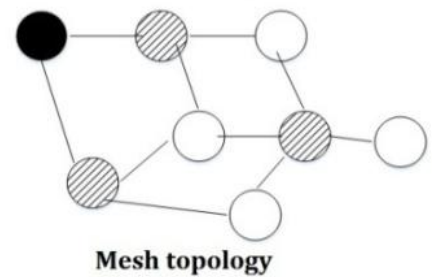

Figure 2. Network topologies

According to the running situation of the city bus, the running route of the bus is fixed. Bus stops are neatly arranged on both sides of the road with obvious distribution law and is very suitable for the Tree topology. Schematic diagram of ZigBee node layout is shown in Figure 3. The figure is only a schematic diagram. The actual layout of the ZigBee needs to be carefully planned and arranged according to the actual situation of the city. In the figure, the adjacent ZigBee nodes can communicate with each other, but the coordinator node is also responsible for the communication with the bus control center [5]. Thus, the bus information can be transmitted to the bus stop node through ZigBee. Due to the relay between each bus stop, the signal will reach the nearest coordinator node, and the coordinator node can send the message to the bus control center directly.
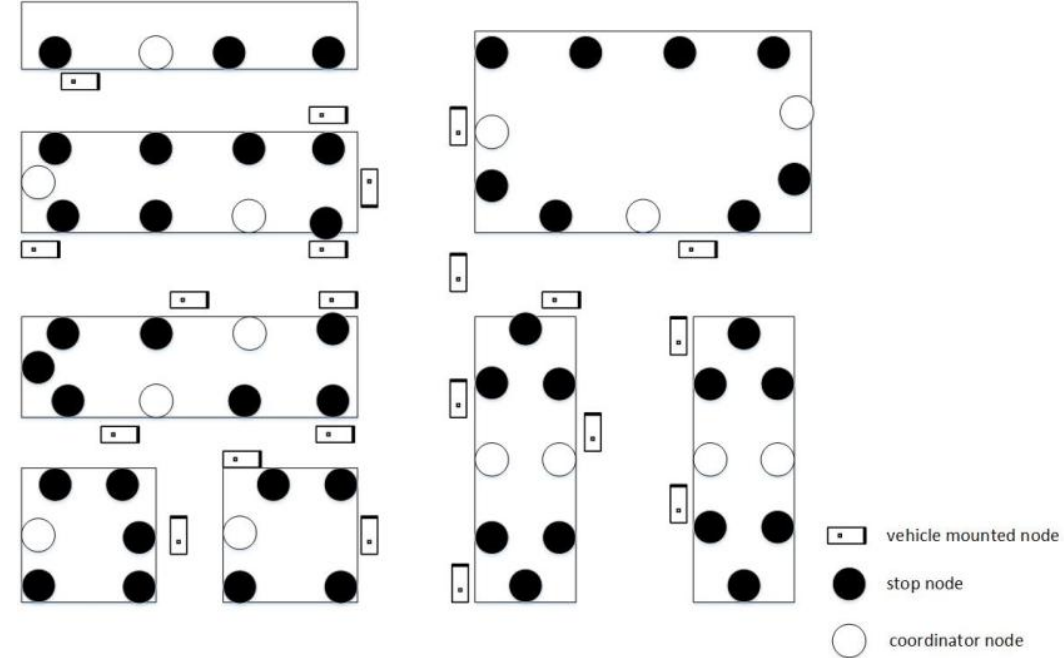

Figure 3. Node layout 


\subsection{Research and design of intelligent public transportation adaptive directional routing algorithm}

Except the bus node, nodes in intelligent public transportation ZigBee communication network all have the ability to route. When a node has both routing capability and rout discovery capability, the node can be used as a routing node [2]. Routing capability is the ability to use a routing table to establish a route through the coordinator or routing node relay to reach a specific destination node. Route discovery table refers to the address information of other nodes broadcasting, which exist in the routing node or the coordinator and is maintained by the routing node.

ZigBee route discovery process is followed. The sender node called src and the destination node called dest can be defined. At first src node broadcasts a route request packet to all adjacent nodes. Each node will continue to broadcast this packet and each packet has a value that represents the quality of the routing. When a node passes, the value of packet is added to the link quality of the link. When all the routes have arrived at dest node, dest node will select the path with the minimum link quality value as the optimal path for the packet. After selecting the optimal route, dest node sends a reply to the previous node and the node will update the routing table after receiving the packet. Note that the routing table is indicated from a source node to a destination node, and the packet is then forwarded to the previous node. The packet will be forwarded according to the message recorded in the route discovery table. When it reaches the src node, a route request is completed.

\subsubsection{The routing table}

The routing table is saved by the coordination point or the routing node, which is used to forward the routing packets, whose items are shown in Table 1.

Table 1 . The routing table

\begin{tabular}{|l|l|l|l|l|l|}
\hline Bits: 16 & 3 & 1 & 1 & 1 & 16 \\
\hline $\begin{array}{l}\text { Destination } \\
\text { address }\end{array}$ & Status & Many-to-one & $\begin{array}{l}\text { Route record } \\
\text { required }\end{array}$ & $\begin{array}{l}\text { Group } \\
\text { ID flag }\end{array}$ & $\begin{array}{l}\text { Next-hop } \\
\text { address }\end{array}$ \\
\hline
\end{tabular}

- Destination address: It is the destination node of this link.

- Status: It is the state information of the link route.

- Many-to-one: It is identifier of coordinator.

- Route record required: It is command frame sequence identifier.

- Group ID flag: It is ID identification of destination network.

- Next-hop address: It is Next-hop node network address for destination node.

\subsubsection{The route discovery table}

The route discovery table is also saved by the coordination point or the routing node, which is used to store some temporary routing information during the route discovery process. Table 2 is a route discovery table.

\begin{tabular}{|c|c|c|c|c|c|}
\hline Bits: 8 & 16 & 16 & 8 & 8 & 16 \\
\hline $\begin{array}{l}\text { Route request } \\
\text { ID }\end{array}$ & $\begin{array}{l}\text { Source } \\
\text { Address }\end{array}$ & $\begin{array}{l}\text { Sender } \\
\text { Address }\end{array}$ & $\begin{array}{l}\text { Forward } \\
\text { Cost }\end{array}$ & $\begin{array}{l}\text { Residual } \\
\text { Cost }\end{array}$ & $\begin{array}{l}\text { Expiration } \\
\text { Time }\end{array}$ \\
\hline
\end{tabular}

- Route request ID: It is the serial number of the route request command frame, which is determined according to the number of routing requests.

- $\quad$ Source Address: It is the network address of the routing request.

- Sender Address: It is the network address of the node which sends the routing request.

- Forward Cost: It is the cost of this routing path.

- Residual Cost: It is the cumulative routing cost of the current node to the destination node.

- Expiration Time: It is the subtraction timer indicates the number of milliseconds until the termination of the route discovery.

\subsubsection{Functions of the routing node}

In the whole intelligent public transportation based on ZigBee communication system, the routing node should have the following functions: 
- Forward information: Getting the message from the bus to the remote information processing center in the network, which acts the role of forwarding.

- Select the most reasonable route to guide the communication: The routing table lists the address of each node in the network, as well as the path between the nodes and the transmission costs associated with them. If there is more than one path to a particular node, the optimal path should be selected, which is also the most economical path based on the predefined criteria. Due to a variety of network segments and their mutual connections may change, the routing information needs to be updated, which is updated regularly provided by the routing information protocol used by changes or update. Each router in the network updates its routing table dynamically according to this rule in order to maintain the effective routing information.

- Lead the communication: The main task of the router is to lead the communication to the destination network and then to the specific node station address. The latter function is accomplished through the network address decomposition.

- Broadcast: Broadcast data frames from the top layer.

- Detect fault: The coordination point and routing node should be able to detect fault, determine which nodes are faulty, start end to end routing repair, and start the local route repair on behalf of other routing nodes.

\subsubsection{Types of nodes in intelligent public transportation ZigBee network}

Intelligent public transportation ZigBee network contains three types of nodes: The coordination nodes equipped on some electronic bus stop boards, the routing nodes equipped on most of the electronic bus stop boards and the vehicle nodes equipped on buses [4]. The functions of the first two types with routing capabilities are basically the same, so in terms of routing, the coordination node can be equated as the routing node. As a RFD node, the node equipped on the bus itself does not have the ability to route. The 'routing' carried by the bus means when it is moving, it is added to the next routing node network according to the routing table that is stored in its own. Vehicle mounted node routing table is virtually a table that essentially reflects the order of all routing nodes and mobile nodes running on the route where the vehicle node is running, and is different from the real meaning of routing, so this paper mainly studies the rout of routing node.

\subsection{Directional routing algorithm}

Figure 4 shows the distribution of the bus stops on both sides of the city bus lines. Routing nodes are scheduled on the bus stops, so the routing nodes along the bus lines are distributed symmetrically. Due to the limited transmission distance of ZigBee nodes, as shown in Figure 5, a node can only communicate directly with a few nodes around it. For example, the ones who can communicate directly with A5 are routing nodes A4, A6, A14, A15 and A16. Information data is transferred from the routing node A5 to A7 and can select a number of routing paths. Data frame can be forwarded in accordance with the order of A5-A6-A7, and also can be forwarded with the order of A5-A16-A17-A7, or A5-A16-A7, or other alternative routing path for forwarding.

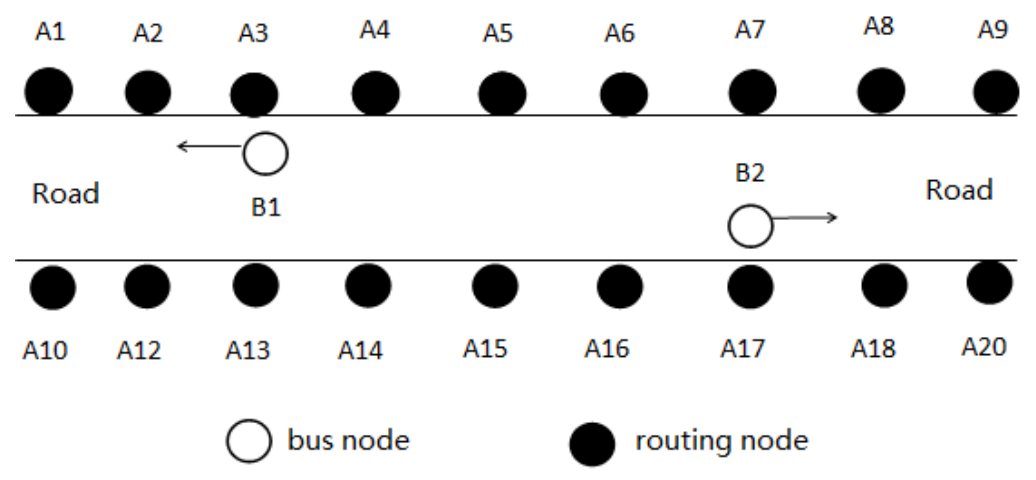

Figure 4. Route layout

To evaluate the routing path, according to the principle of minimum number of hops equal to the lowest path cost, the path cost of rout A5-A6-A7 is obviously the lowest. According to the minimum path cost routing algorithm, bus route node data flow is shown in Figure 6. When the destination node and the sending node are in the same side of the road, the routing path along the side of the road is the lowest cost. When the destination node and the sending node are on different sides of the road, the data will be transmitted to the opposite side of the routing node firstly, and then forwarded along the road to ensure the lowest cost routing. The routing node at the same side as the uplink route of a bus route can be stipulated, and the other side as the downlink route. The forwarding and flow of data between the routing nodes is consistent with the direction of the 
bus route, and the direction of the flow of data is regular and the direction is determined. So, it can be called 'directional routing algorithm'. Directional routing algorithm in intelligent public transportation network is such that the routing of data transfer should try to use a directed line and avoid situations where the data transfer from the uplink route to the downlink route, and then is sent back to the uplink route. For example, the routing node A4 receives a frame of data, whose destination node is routing node A8. Then, in accordance with the directional routing algorithm, data can only be forwarded along the path A4-A5-A6-A7-A8. As shown in Figure 6, when some nodes in the A4-A5-A6-A7-A8 routing path fail to perform the routing function, the minimum routing cost we should select is the routing path A4-A15-A16-A17-A8. But, there is a problem in such a route. Because the distance between A4 and A15 is a little far away, the ZigBee signal is weak, and it will not be stable. A large number of such ZigBee links will cause the entire network to be complex, and there may be unforeseen problems that appear. Therefore, when the same side route can't be used normally, the forwarding of the routing node will first be sent to the routing node on the opposite side and then be forwarded. So, the routing path from A4 to A8 is changed to A4-A14-A15-A16-A17-A18-A8. When both opposite side routing nodes occur fault, such a routing path like A4-A15-A16A17-A8 can be used. The directional routing algorithm avoids the phenomenon of data routing cycle, and the algorithm is simple and easy to implement.

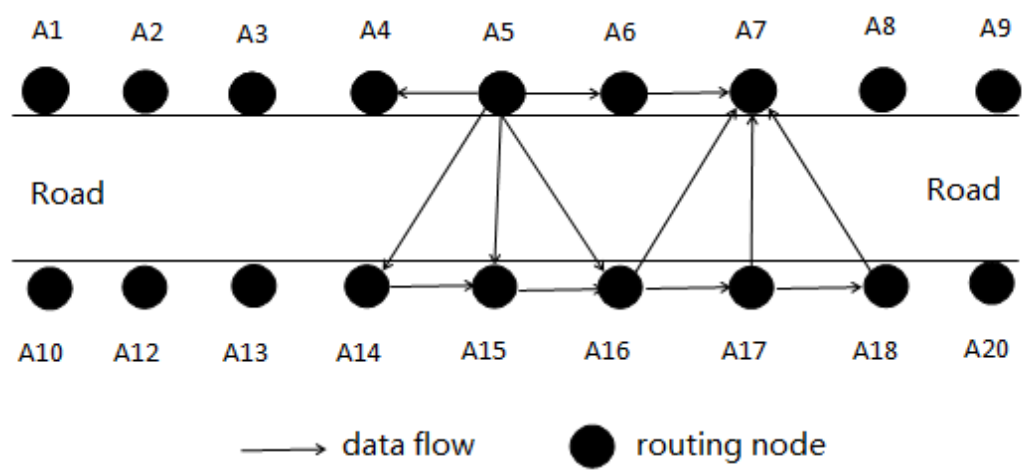

Figure 5. System communication
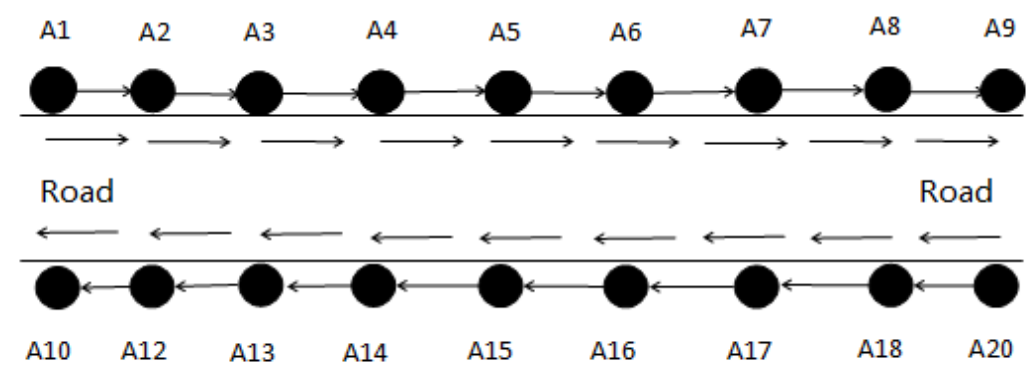

data flow routing node

Figure 6. Directional routing

\subsection{Realization of intelligent public transportation adaptive directional routing}

First, when the routing node receives a data frame, no matter if it is from the top layer or bottom layer, it needs to judge whether it is a broadcast frame. If it is a broadcast frame, it broadcasts it directly. If it is not a broadcast frame, it is necessary to determine whether the destination address of the frame is the node itself. If it is, the data will be sent to the bottom layer, the information processing center. If it is not, the routing address will be resolved and see if the network address exists. If it does not exist, the route discovery is started to look for the destination node of the frame. If it exists, it is needed to judge if the routing node is on the same side. If it is on the same side, we need to determine whether the next hop is normal. If it is normal, the frame is forwarded to the next hop on the same side, otherwise it determines whether the other side routing node is normal. If it is normal, data is forwarded to the routing node, otherwise to other paths. If the destination address of the frame is located on a different side, it is determined whether the routing node on the different side is normal. If it is normal and has the forwarding capacity, the frame will be forwarded to the route on the other side. If the routing node that is on the other side doesn't have the ability to route for some reason, then choose other available routing nodes to forward. 


\section{Scheme of bus positioning}

Bus drivers are familiar with the bus routes and the traffic situation in the city, so the significance of positioning is not navigation. No matter the positioning, bus running information must be provided to the bus control system, or to provide the estimated time of the bus to citizens. The positioning information of the bus is not required to be accurate. So, the positioning based on ZigBee only needs to give the general positioning of the bus.

In this paper, the bus positioning technology is based on the ZigBee RSSI positioning technology [6]. In general, the energy, computing power and storage capacity of ZigBee nodes are limited. It needs low complexity computation algorithm and low communication overhead. ZigBee communication protocol carries the RSSI information. Positioning based on RSSI can be easy to achieve without additional hardware equipment, and it is low power and low cost.

The loss model of radio propagation path for free space is shown as followed.

$$
P L(d)=32.44+10 n \lg d+10 n \lg f
$$

In Equation (1), $\mathrm{d}$ is the distance between the receiver and the transmitter $(\mathrm{km}), \mathrm{f}$ is the radio frequency $(\mathrm{MHz})$, and $\mathrm{n}$ is the path attenuation factor whose value is between 2 and 5.

In the practical application environment, due to multipath, diffraction and obstacle factors, the loss of radio propagation path will be different from the theoretical value. It often uses a log normal distribution model and the following Equation (2) is the mathematical expression of the model.

$$
P L(d)=P L\left(d_{0}\right)+10 n \lg \left(d / d_{0}\right)+X_{\sigma}
$$

In Equation (2), PL(d) is the loss of the distance of d, and d0 is the reference distance whose value usually is $1 \mathrm{~m}$. $X_{\sigma}$ is a Gauss distribution random variable with average value of 0 , and the standard deviation usually varies from 4 to 10 . PL (d0) is the path loss after reference distance of $\mathrm{d} 0$. The signal intensity of the receiver can be obtained by Equation (1) and Equation (2), which is given by the following equation.

$$
\operatorname{Pr}(d)=P t-P L(d)
$$

In Equation (3), Pr(d) is the signal strength of the receiver, which can also be expressed as RSSI(d). Pt is the signal power of transmitter. Based on the principle above, IEEE802.15.4 gives a simplified channel model, which is given by the following Equation (4).

$$
\operatorname{RSSI}(d)= \begin{cases}P_{t}-40.2-10 \times 2 \times \lg d & d \leq 8 m \\ P_{t}-58.5-10 \times 3.3 \times \lg d & d>8 m\end{cases}
$$

Experiments show that the relationship between RSSI and d is shown in Figure 7. It can be seen that when the distance is more than $100 \mathrm{~m}$, the signal enhancement of ZigBee descends gently. But, when the distance is within the $100 \mathrm{~m}$, the range of the signal strength will be significant, and it shows the status of jumping.

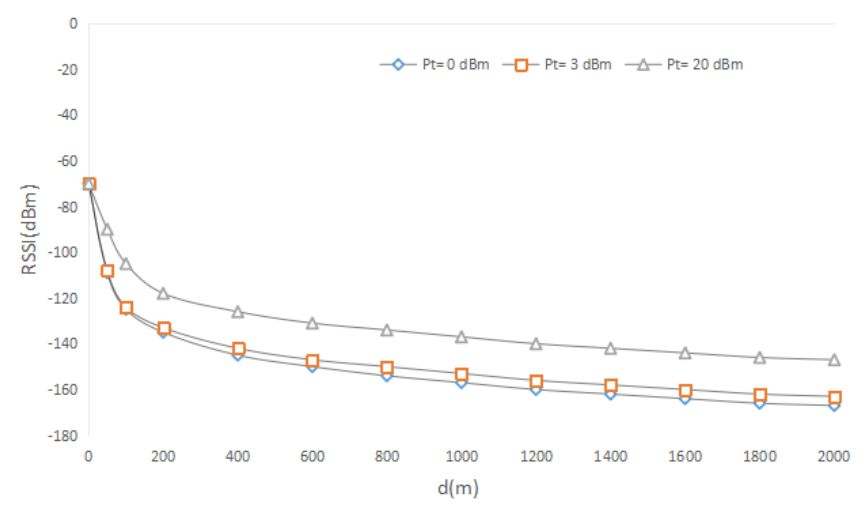

Figure 7. Relationship between RSSI and d 
ZigBee signal source is generally arranged on the bus stop, so the mutation of the signal will appear when the bus is close to the bus stop or left the bus stop. So, the positioning of the bus in the bus routes can be divided into two cases. One positioning is near the bus stop, and another is between two bus stops.

We can divide the regions near the bus stop into three parts, such as the $40 \mathrm{~m}$ when the bus is near the bus stop, the $20 \mathrm{~m}$ when the bus stops, and the 40m when the bus leaves the bus stop. In these three regions, compared with other bus stop boards, the signal intensity received by the bus from the bus stop board is obviously different. We can only use the stop signal source to position, which is called single signal source positioning. In this case, when the signal received by the bus varies from weak to strong and gradually reaches a certain value, we can think the bus is arriving. The arrival station and positioning information can be analyzed from the ZigBee signal of the stop. When the bus leaves the stop, the received signal will gradually weaken until a value, then it can be considered to have left the stop. Another case is the positioning between two bus stops. In this case, the bus node will receive signals from several stops, and the intensity of each signal source does not appear too big of a gap, then we can use the trilateration positioning method to collect the strongest three signals of all.

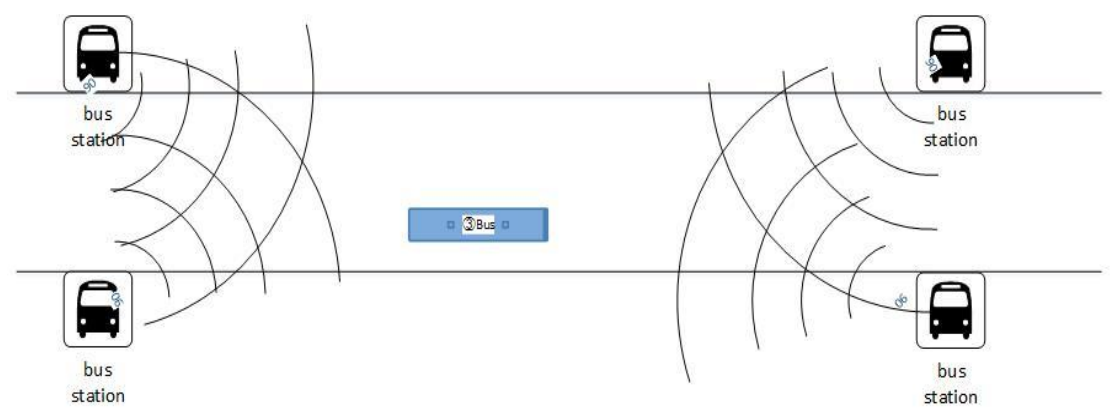

Figure 8. Trilateration positioning

As shown in Figure 8, the bus can receive the signals from the bus stop A, B, C and D. Coordinates of the four stops are known, and they broadcast through ZigBee. Assume the coordinate of A stop is $\left(X_{a}, Y_{a}\right), B$ stop is $\left(X_{b}, Y_{b}\right), C$ stop is $\left(X_{c}, Y_{c}\right)$, and D stop is $\left(\mathrm{X}_{\mathrm{d}}, \mathrm{Y}_{\mathrm{d}}\right)$. Among them, the signals of A, B and C are strong, and the signal intensity is respectively RSSI (A), RSSI (B) and RSSI (C). According to Equation (4), distance between the bus node and the bus stop A, B and C can be calculated respectively as $d_{a}, d_{b}$ and $d_{c}$, then use the trilateration method to get the following Equation (5).

$$
\left\{\begin{array}{l}
\left(x-X_{a}\right)^{2}+\left(y-Y_{a}\right)^{2}=d^{2}{ }_{a} \\
\left(x-X_{b}\right)^{2}+\left(y-Y_{b}\right)^{2}=d^{2}{ }_{b} \\
\left(x-X_{c}\right)^{2}+\left(y-Y_{c}\right)^{2}=d^{2}{ }_{c}
\end{array}\right.
$$

From Equation (5), we can obtain the coordinates of the vehicle node M1 (x, y) as the following Equation (6).

$$
\left[\begin{array}{l}
x \\
y
\end{array}\right]=\left[\begin{array}{l}
2\left(X_{a}-X_{c}\right) 2\left(Y_{a}-Y_{c}\right) \\
2\left(X_{b}-X_{c}\right) 2\left(Y_{b}-Y_{c}\right)
\end{array}\right]^{-1}\left[\begin{array}{c}
X^{2}{ }_{a}-X^{2}{ }_{c}+Y^{2}{ }_{a}-Y^{2}{ }_{c}-d^{2}{ }_{a}+d^{2}{ }_{c} \\
X^{2}{ }_{b}-X^{2}{ }_{c}+Y^{2}{ }_{b}-Y^{2}{ }_{c}-d^{2}{ }_{b}+d^{2}{ }_{c}
\end{array}\right]
$$

Some errors will exist in such positioning method mainly because the signal of stop node is not stable, and the vehicle itself and the external interference will produce large fluctuations with RSSI value additionally. In order to eliminate this kind of fluctuation and make the positioning as accurate as possible, we can compare the ZigBee positioning results with the last positioning coordinates. If the difference exceeds a certain amount, the positioning results can't be considered trusted. We should lead into a historical positioning for correction, give up the current results and calculate a prediction results with the prior information as substitution. This can improve the positioning accuracy of the intelligent public transportation system based on ZigBee to a certain extent.

\section{Design of intelligent electronic bus stop board}

Intelligent electronic bus stop board is an important part of the system. It can be transformed based on the most common bus stop. It does not need to set up any other lines or external power supply. It just uses a small piece of solar panel to make the bus stop board operate normally. The wireless communication between electronic bus stops and that between the electronic bus stop board and bus is based on ZigBee module. On the electronic board, information notification can be expressed clearly 
and only needs to use several LED lamps, which will greatly save energy. Smart phones can also receive the broadcast information of the bus stop. Some more detailed information of the bus can be seen by some apps to meet the needs of different people. Because people only care about the information of the next bus, the design of intelligent electronic bus stop board can refer to Figure 9. For example, there are three bus routes on the electronic bus stop board of the $\mathrm{Zz}$ bus station including line 315 , line 316 and line 317, and some passengers need to take line 315. In line 315, the bottom of each station before Zz station has set a LED indicator lamp, which is used to indicate which station the bus has just arrived at. As shown in Figure 9, the next bus in line 315 has arrived at Xx station. If the time for each bus station is about 3 minutes, and there are two stations from $\mathrm{Xx}$ station to $\mathrm{Zz}$ station, according to this information, the passengers will be able to estimate that the next bus will arrive in about 6 minutes. Similarly, passengers waiting for line 316 can also get the information from the bus stop that the next bus in line 316 will arrive in 3 minutes.

Because the route used in intelligent public transportation system based on ZigBee is directional routing, the realization of the bus stop board is quite easy. When a bus arrives at the station, the information will be transferred along the bus route, and the next few bus stops on the bus route can capture the information. Then, the information is displayed through the LED lamp, and the function of intelligent electronic bus stop board is realized.

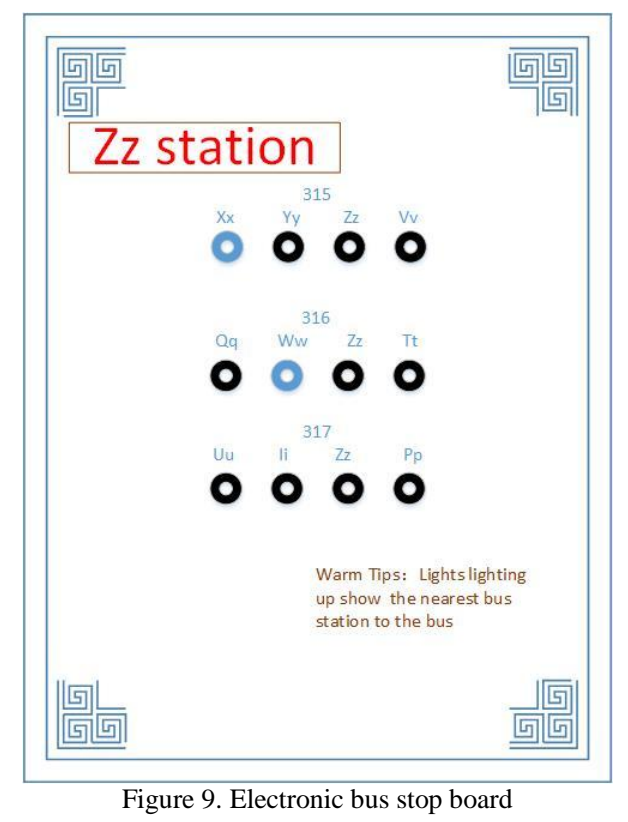

\section{Conclusions}

ZigBee is a kind of technology that is widely used in the Internet of Things(IoT). It opens a better way for the construction of intelligent public transportation system. Its inherent low cost, self-networking and other advantages make the construction of intelligent public transport able to be extended to a large extent, but not limited to economically developed cities. The intelligent public transportation system based on ZigBee technology proposed in this paper can realize the positioning of busses, information transmission, routing selection, intelligent electronic bus stop boards and other basic functions, which will have reference function to the development of intelligent transportation systems.

\section{Acknowledgments}

My deepest gratitude goes first and foremost to Associate Professor Xiuxia Yu for her encouragement and guidance. I am also greatly indebted to my beloved family for their loving considerations and confidence in me. Last but not least, I owe much to my friends for their valuable suggestions and critiques, which are of great help and importance in making this thesis a reality.

\section{References}

1. H. F. Han, K. M. Du, Z. F. Sun, W. Zhao, R. Chen, and J. B. Liang, "Design and Application of ZigBee Based Telemonitoring System for Greenhouse Environment Data Acquisition," Transactions of the Chinese Society of Agricultural Engineering, vol.25, 
no.7, pp.158-163, 2009

2. S. B. Jiao, D. Song, Q. Zhang, and J. W. Tang, "Coal Mine Monitoring System Based on ZigBee Wireless Sensor Networks," Journal of Electronic Measurement and Instrumentation, vol.27, no.5, pp.436-442, 2013

3. J. B. Li, and Y. Z. Hu, "Design of ZigBee Network Based on CC2530," Electronic Design Engineering, vol.19, no.16, pp.108111,2011

4. M. Li, R. Wang, and L. Shi, "Wireless Sensors Network Node Based on ZigBee," Techniques of Automation and Applications, vol.27, no.1, pp.91-94, 2008

5. Z. H. Qian, S. Zhu, and X. Wang, "An Cluster-Based ZigBee Routing Algorithm for Network Energy Optimization," Chinese Journal of Computers, vol.36, no.3, pp.485-493, 2013

6. X. Shi, A. M. Yin, and X. Chen, "RSSI and Multidimensional Scaling Based Indoor Localization Algorithm," Chinese Journal of Scientific Instrument, vol.35, no.2, pp.261-267, 2014

7. J. Zhan, and W. Shi, "Design of Public Transport Communicate Wireless Network Based on ZigBee," Modern Electronics Technique, vol.30, no.10, pp.118-120, 2007

8. K. Y. Zhu, J. Y. Liu, Y. L. An, W. Y. Wang, and F. Y. Wang, "City Intelligence Public Transportation Network System Based on ZigBee," Microcontrollers \& Embedded Systems, pp.17-20, 2008 